\title{
CALCULATED POWDER X-RAY DIFFRACTION DATA FOR BORON
}

C. E. Holcombe, Jr

A. L. Coffey, Jr
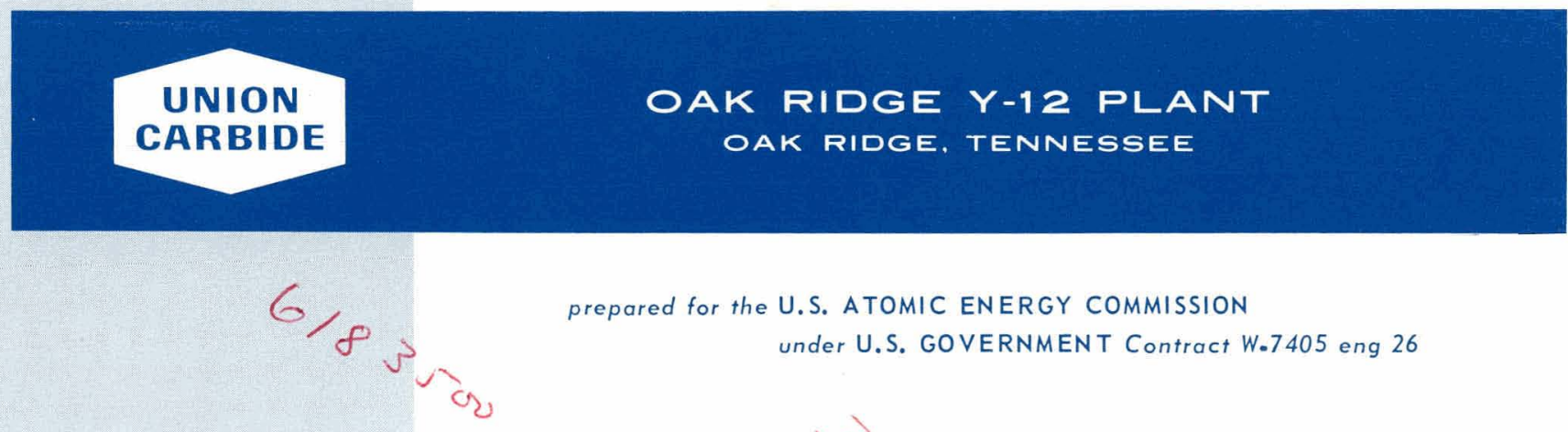

prepared for the U.S. ATOMIC ENERGY COMMISSION

under U.S. GOVERNMENT Contract W.7405 eng 26

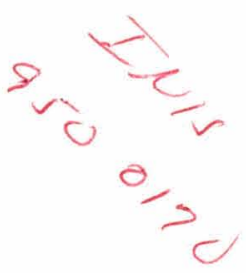




\section{DISCLAIMER}

This report was prepared as an account of work sponsored by an agency of the United States Government. Neither the United States Government nor any agency Thereof, nor any of their employees, makes any warranty, express or implied, or assumes any legal liability or responsibility for the accuracy, completeness, or usefulness of any information, apparatus, product, or process disclosed, or represents that its use would not infringe privately owned rights. Reference herein to any specific commercial product, process, or service by trade name, trademark, manufacturer, or otherwise does not necessarily constitute or imply its endorsement, recommendation, or favoring by the United States Government or any agency thereof. The views and opinions of authors expressed herein do not necessarily state or reflect those of the United States Government or any agency thereof. 


\section{DISCLAIMER}

Portions of this document may be illegible in electronic image products. Images are produced from the best available original document. 


\begin{tabular}{|c|}
\hline Printed in the United States of America. Available from \\
National Technical Information Service \\
U.S. Department of Commerce \\
5285 Port Royal Road, Springfield, Virginia 22151 \\
Price: Printed Copy $\$ 4.00$; Microfiche $\$ 0.95$
\end{tabular}

This report was prepared as an account of work sponsored by the United States Government. Neither the United States nor the United States Atomic Energy Commission, nor any of their employees, nor any of their contractors, subcontractors, or their employees, makes any warranty, express or implied, or assumes any legal liability or responsibility for the accuracy, completeness or usefulness of any information, apparatus, product or process disclosed, or represents that its use would not infringe privately owned rights. 


\section{CALCULATED POWDER X-RAY DIFFRACTION DATA FOR BORON}

C. E. Holcombe, Jr
A. L. Coffey, Jr

Oak Ridge Y-12 Plant

P.O. Box Y, Oak Ridge, Tennessee 37830

Date Issued - May 25, 1973

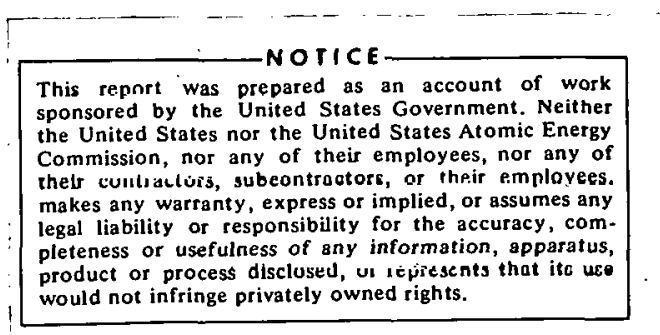

Prepercd for the U.S. Atomic Energy Commissinn Under U.S. Government Contract W.7405eng.26 


\section{ABSTRACT}

A literature review has been made of the crystallographic data, and a study has been made of computer-simulated powder $\mathrm{X}$-ray diffraction data and of the solid solution formation for boron polymorphs. 


\section{CONTENTS}

SUMMARY $\ldots \ldots \ldots \ldots \ldots \ldots \ldots \ldots \ldots \ldots \ldots \ldots$

INTRODUCTION ..................... 5

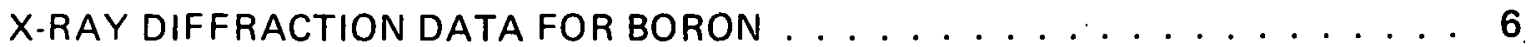

Literature Review . . . . . . . . . . . . . . . . . . 6

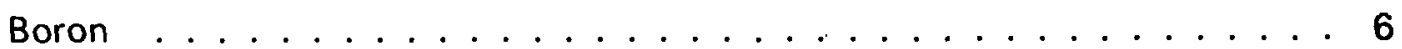

Solid Solutions with Boron $\ldots \ldots \ldots \ldots$

Computer Simulation $\ldots \ldots \ldots \ldots \ldots \ldots \ldots \ldots \ldots$

Powder Diffraction Data $\ldots \ldots \ldots \ldots \ldots$

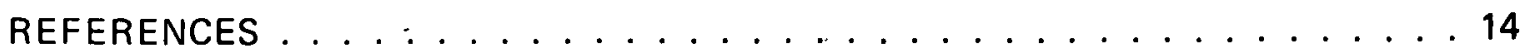




\section{SUMMARY}

Pertinent literature involving boron and its solid solutions was reviewed. The powder $X$-ray diffraction patterns were computed for the three forms of boron for which refined crystal parameters are available: $a$-rhombohedral, $\beta$-rhombohedral, and $a$-tetragonal. The data include reflections for both CuKa1-2 and $\mathrm{CrKa}_{1-2}$ up to 145 degrees $2 \theta$, and both integrated and peak-height intensities are listed. The solid solutions which can occur with these forms of boron are discussed in terms of the interstitial and substitutional types which can occur. 


\section{INTRODUCTION}

An understanding of the crystallography of boron and an improved understanding of its associated powder diffraction data should be a beneficial aid to predicting the behavior of its compounds and solid solutions.

Although much effort has been devoted in the literature to the preparation and characterization of compounds of boron, the element itself has not been studied in depth. Relationships of the various forms of boron and their solid solutions with other elements have been discussed in many separate papers, and the appropriate powder X-ray diffraction data are scattered throughout the literature and contain many deficiencies although the crystal structures have been studied in detail. It is the purpose of this study: (1) to combine all the pertinent crystallographic data necessary for studying boron and its solid solutions; (2) to present computer-simulated (from reported single-crystal data) X-ray powder diffraction patterns for the forms of boron; (3) to illustrate the utility of obtaining computer-simulated patterns, and (4) to discuss possible solid solutions with boron. 


\section{X-RAY DIFFRACTION DATA FOR BORON}

\section{LITERATURE REVIEW}

\section{Boron}

Boron has been shown ( 1 - 3) to exist in three distinct polymorphic forms: a-rhombohedral, $\beta$-rhombohedral, and a-tetragonal. Of the several other polymorphic forms proposed, another tetragonal form (4) may eventually be shown to be a true form of boron. Thus, the known tetragonal form is properly designated as " $a$-tetragonal". The $a$-rhombohedral form occurs from the decomposition of boron iodide or hydrides in the temperature range from 800 to $1,200^{\circ} \mathrm{C}$ on various substrates. The $\beta$-rhombohedral form appears to be the thermodynamically stable polymorph and is consistently produced at $1,5000 \mathrm{C}$ or above. A study of the $a$-to- $\beta$ transformation in boron has recently been published.(5) The $a$-tetragonal form is produced from the reduction of boron bromide with hydrogen on a hot filament (tungsten or tantalum) at 1,100 to $1,300^{\circ} \mathrm{C}$. Each of these three forms ( $a$-rhombohedral, $\beta$-rhombohedral, and $a$-tetragonal) is dependent on icosahedral packing and has its own respective structural designation: $B_{12} ;(B 84)\left(B_{10}\right)_{2} B_{\text {; }}$ and $\left(B_{12}\right)_{4} B_{2}$. The $B 84$ unit consists of a central icosahedron with 12 half-icosahedra (or pentagonal pyramids) surrounding it in icosahedral symmetry.

The powder diffraction pattern, using CuKa radiation, published for a-rhombohedral boron(1) was not calculated from single-crystal data, and the reported d-spacings are consistently shifted when compared to those calculated from the refined lattice parameters.

The previous patterns for $\beta$-rhombohedral and $a$-tetragonal boron $(6)$ included only the data for the CrKa radiation. Many weak peaks were not included; and, thus, comparison with solid solutions containing dramatic intensity differences could be difficult using only the reported data. Also, their $\beta$ rhombohedral pattern was presumably hased on the best refinement of that structure in 1960 . Later data $(2,7)$ used in the present calculations included the least-squares refined positional coordinates and the partial occupancy factors. Several intensity differences occurred, compared with data reported by Hoard and Newkirk. (6)

\section{Solid Solutions with Boron}

When the basic framework of the parent material, boron, is unaltered upon injection of interstitial or substitutional atoms into the structure, the resultant material should properly he colled a colid solution. Solid snlutions with $a$-rhombohedral boron, $(8-11)$ $\beta$-rhombohedral boron, $(12-14)$ and $a$-tetragonal boron(15) have been reported to exist . with, in many cases, minimal changes in the lattice parameters and significant changes in intensities of the diffracting planes.

Interstitial derivatives of a-rhombohedral boron are formed when Positions $1 \mathrm{~b}$ or $2 \mathrm{c}$ of Space Group $R \overline{3} m$ are filled with atoms such as boron, carbon, oxygen, aluminum, silicon, phosphorus, sulfur, or arsenic. These sites may be either partially or completely filled. Silicon has been shown (11) to substitute for boron atoms in the icosahedra as well as in the interstitial sites. Alpha tetragonal boron has interstitial sites at Positions 2a, 4a, and 4c 
which can easily accommodate foreign atoms. Smaller atoms (beryllium, carbon, and aluminum), according to Matkovich, et al, (15) can fill either the $4 \mathrm{a}$ or $4 \mathrm{c}$ sites. Larger atoms (such as tantalum or tungsten) can be inserted into the $2 a$ sites up to a limit of 3.8 atom percent of the impurity atom, according to Hoard and Newkirk. (6)

In $\beta$-rhombohedral boron, Sullenger (7) listed the various holes in the structure suitable for interstitial atoms. The radius of the largest atoms which could be inserted was $1.18 \mathrm{~A}$ (two available sites), and eight other sites occurred which would accommodate atoms with radii greater than 1.0 A. These sites could be occupied by such atoms as aluminum, silicon, arsenic, and phosphorus, as well as smaller atoms. A substitutional solid solution(12) of smaller atoms for some of the 21 boron atoms which are not included in the B84 polyhedral units is also possible. This situation apparently occurs with silicon and aluminum atoms. Also, larger transition elements may substitute in the $2 c$ sites for the two $B_{10}$ units. Other substitutional variants have been discussed by Anderson and Lundstrom. (14) These variants would explain the solid solutions with boron described by Carlsson and Lundstrom (13) for many transition elements ( $\mathrm{Sc}, \mathrm{Ti}, \mathrm{V}, \mathrm{Cr}, \mathrm{Mn}, \mathrm{Fe}, \mathrm{Co}, \mathrm{Ni}$, and $\mathrm{Cu}$ ) up to 4.8 atom percent. Also, a similar solid solution occurred with zinc up to 4.2 atom percent dissolved in boron(16) under gas pressure autuclaving. These transition elements caused small or no changes in the lattice parameters, but caused substantial intensity variations. These changes in the diffraction pattern combined with possible superlattice lines from ordered solid solutions may be responsible for the many reported polymorphs of boron.

\section{COMPUTER SIMULATION}

Calculation of a complete set of integrated intensity values for a random powder sample of a crystalline material can be readily accomplished with a computer program developed by Smith. $(17,18)$ If the input structure data are of high quality, the integrated intensities match very closely with the experimentally observed data. This degree of fit justifies the use of such calculated patterns as standards for quantitative phase analysis as well as identification.

The fundamental equation used by Smith's program in the calculation of the set of integrated intensity values from a specific atomic arrangement and unit cell size is:

$$
1=\text { ap LPAT }|F|^{2}
$$

where "a" represents a scale factor used to place the data on a relative scale with a maximum of 100, " $p$ " the multiplicity factor, "LP" the Lorentz-polarization factor, "A" the absorption factor, " $T$ " the Debye temperature factor, and " $F$ " the structure factor.

The necessary input data include the unit cell parameters, the space group, the atom position parameters and their scattering and temperature factors, the mass absorption coefficient and crystal density, and the $X$-ray wavelength used. Other useful data include the extinction conditions and the Miller indices of the strongest reflection. Using the space group and the unit cell constants, the full set of nonredundant indices and d-spacings is generated. Reflections not allowed by space-group extinction are eliminated to minimize calculations. Integrated intensities for each $\mathrm{HKL}$ are then generated by calculating the temperature-modificd structure factor using the appropriate multiplicity factor and 
Table 1

CRYSTAL CATA USED FOR BORON

\begin{tabular}{|c|c|c|}
\hline a-Rhombohedral Boion & $\beta$-Rhombohedral Boron & a-Tetragonal Boron \\
\hline Reference: Decker and Kasper $\{1950\}$ & $\begin{array}{l}\text { References: Hoard, Sullenger, Kennard, } \\
\text { and Hughes (1970), and Sullenger (1969) }\end{array}$ & Reference: Hoard, Hughes, and Sands (1958) \\
\hline Space Group: $R \overline{3} \mathrm{~m}(166)$ & Space G:cup: R $\overline{3} \mathrm{~m}$ (166) & Spaze Group: P42 nnm (134) \\
\hline Crystal System Rhombohedrall & Crysal System: Rhombohedral & Crystal System: Tetragonal \\
\hline $\begin{array}{l}\text { Rhombohedral Lattice Parameters: } \\
a_{0}=5.057 \pm 0.003 A_{;} a=58.06 \pm 0.05^{\circ}\end{array}$ & $\begin{array}{l}\text { Rhombohedral Lattice Parameters: } \\
a_{0}=10.145 \pm 0.015 \mathrm{~A} ; a=65.28 \pm 0.13^{\circ}\end{array}$ & $\begin{array}{l}\text { Lattice Parameters: } \\
a_{0}=8.74 \pm 0.015 A ; c_{0}=5.068 \pm 0.010 A\end{array}$ \\
\hline $\begin{array}{l}\text { Hexagonal Latlice Parameters: } \\
a_{0}=4.908 \pm 0.003 \mathrm{~A} ; c_{0}=12.567 \doteq 0.007 \mathrm{~A}\end{array}$ & $\begin{array}{l}\text { Hexagonal Lattice Parameters: } \\
a_{O}=10.944 \pm 0.015 A ; c_{0}=23.81 \pm 0.03 \mathrm{~A}\end{array}$ & \\
\hline Crystal Density: $2.46 \mathrm{gms} / \mathrm{cc}$ & Crystal Density: $2.29 \mathrm{gms} / \mathrm{cc}$ & Crystal Density: $2.33 \mathrm{gms} / \mathrm{cc}$ \\
\hline Structural Designation: $\mathbf{B}_{12}$ & Struztural Designation: $\left(\mathrm{B}_{84}\right)\left(\mathrm{B}_{10}\right)_{2} \mathrm{~B}$ & Structural Designation: $\left(\mathrm{B}_{12}\right)_{4} \mathrm{~B}_{2}$ \\
\hline Number Atoms in Unit Cell: 12 & Number Atoms in Unit Cell: 105 & Number Atoms in Unit Cell: $\mathbf{5 0}$ \\
\hline
\end{tabular}


applying the Lorentz polarization and absorption factors. Finally, a scale factor is introduced to reduce the calculated intensities to values with a usual maximum of 100 . The numbers obtained are relative integrated intensities and are directly comparable with the total area under a diffractometer profile.

\section{POWDER DIFFRACTION DATA}

The most refined data available on the three forms of boron were used as input for the computer code. The data are listed in Table 1. The references given also contain the atomic positional parameters. For the rhombohedral boron polymorphs, hexagonal parameters were used to generate the diffraction patterns.

The calculated powder diffraction data for $a$-rhombohedral, $\beta$-rhombohedral, and a-tetragonal boron are reported in Tables 2 through 4 , respectively.

Table 2

SIMULATED POWDER DIFFRACTION DATA FOR $a$-RHOMBOHEDRAL BORON

\begin{tabular}{|c|c|c|c|c|c|c|c|c|c|}
\hline \multirow[b]{2}{*}{$\begin{array}{l}\text { d-Spacings } \\
\text { (A) }\end{array}$} & \multirow[b]{2}{*}{$\mathrm{HKL}$} & \multicolumn{2}{|c|}{$\begin{array}{c}\text { CuKa1-2 } \\
\text { Relative Intensities } \\
\text { Flat Absorption } \\
\end{array}$} & \multicolumn{2}{|c|}{$\begin{array}{c}\text { CrKa1-2 } \\
\text { Relative Intensities } \\
\text { Flat Absorption } \\
\end{array}$} & \multirow[b]{2}{*}{$\begin{array}{l}\text { d-Spacings } \\
\text { (A) }\end{array}$} & \multirow[b]{2}{*}{ HKL } & \multicolumn{2}{|c|}{$\begin{array}{c}\text { CuKa1-2 } \\
\text { Relative Intensities } \\
\text { Flat Absorption }\end{array}$} \\
\hline & & Integrated & $\begin{array}{c}\text { Peak } \\
\text { Height }\end{array}$ & Integrated & $\begin{array}{c}\text { Peak } \\
\text { Height }\end{array}$ & & & Integrated & $\begin{array}{c}\text { Peak } \\
\text { Height }\end{array}$ \\
\hline 4.189 & 003 & 55 & 52 & 55 & 56 & \multirow{2}{*}{1.048} & 042 & 21 & \multirow{2}{*}{1} \\
\hline 4.026 & 101 & 100 & 100 & 100 & 100 & & $\{0012$ & $<1\}$ & \\
\hline 3.521 & 012 & 4 & 4 & 4 & 4 & \multirow{2}{*}{1.007} & $40 \overline{4}$ & \multirow{2}{*}{\multicolumn{2}{|c|}{$2\}$}} \\
\hline 2.526 & 104 & 65 & 57 & 61 & 52 & & $\{2011$ & & \\
\hline 2.454 & 110 & 11 & 10 & 10 & 8 & 0.9945 & $03 \widehat{9}$ & 5 & 3 \\
\hline 2.163 & 015 & 4 & 3 & 3 & 3 & 0.9854 & 137 & 1 & $<1$ \\
\hline 2.117 & 113 & 4 & 4 & 4 & 3 & \multirow{2}{*}{0.9426} & $\{318$ & \multirow{2}{*}{\multicolumn{2}{|c|}{2}} \\
\hline 2.095 & 021 & 54 & 44 & 50 & 38 & & $\{1013$ & & \\
\hline 2.013 & 202 & $<1$ & $<1$ & $<1$ & $<1$ & \multirow{2}{*}{0.9313} & s $32 \overline{4}$ & 18 & \multirow{2}{*}{10} \\
\hline 1.654 & 107 & 6 & 5 & 7 & 4 & & $\{1211$ & $4\}$ & \\
\hline 1.623 & 205 & 12 & 9 & 13 & 8 & 0.9217 & $22 \overline{9}$ & $<1$ & $\ll 1$ \\
\hline \multirow[b]{2}{*}{1.594} & 211 & $11)$ & \multirow{2}{*}{9} & $12\}$ & \multirow[b]{2}{*}{8} & 0.9144 & 407 & $\ll 1$ & $\ll 1$ \\
\hline & 116 & $\ll 1\}$ & & $<1\}$ & & 0.9091 & 235 & 2 & 1 \\
\hline 1.473 & 018 & 10 & 7 & 14 & 8 & \multirow{2}{*}{0.9056} & $\{413$ & $2\}$ & \multirow{2}{*}{1} \\
\hline 1.430 & 214 & 24 & 17 & 36 & 19 & & $\{143$ & $<.1\}$ & \\
\hline 1.417 & 300 & 11 & 8 & 18 & 9 & \multirow{2}{*}{0.8800} & 048 & 21 & \multirow{2}{*}{2} \\
\hline 1.396 & 009 & 6 & 4 & 10 & 5 & & $\{0213$ & $3\}$ & \\
\hline 1.371 & 027 & 13 & 9 & 24 & 11 & 0.8598 & $13 \overline{10}$ & 2 & $<1$ \\
\hline 1.353 & 125 & 16 & 11 & 32 & 15 & 0.8569 & $32 \overline{7}$ & 8 & 3 \\
\hline \multirow{2}{*}{1.342} & 303 & $9\}$ & 17 & $18\}$ & 24 & \multirow{3}{*}{0.8481} & 051 & $7)$ & \multirow{3}{*}{3} \\
\hline & 1033 & $16\}$ & 17 & $33\}$ & 24 & & $\{146$ & $\ll 1\}$ & \\
\hline 1.263 & 208 & 19 & 13 & 59 & 24 & & 416 & $1)$ & \\
\hline 1.227 & 220 & 5 & 3 & 19 & 7 & & 502 & $\ll 1$, & \\
\hline 1.197 & 217 & $<1$ & $<1$ & & & 0.8422 & 0312 & $9\}$ & 10 \\
\hline \multirow{2}{*}{1.177} & 223 & 4 & 2 & & & & $30 \overline{12}$ & 17) & \\
\hline & $(131$ & 4 & & & & 0.8378 & טuㅣㅡ & 4 & 2 \\
\hline 1.174 & $\{306$ & $\ll 1\}$ & 3 & & & 0.8285 & $23 \overrightarrow{8}$ & 13.) & 5 \\
\hline & 036 & $\leqslant 1 !$ & & & & 0.0200 & $\{211.3$ & $2\}$ & 5 \\
\hline 1.159 & 312 & 1 & $<1$ & & & 0.8269 & 2014 & $\ll 1$ & $\ll 1$ \\
\hline 1.123 & 128 & 1 & $<1$ & & & 08206 & $\{05 \overline{4}$ & $15\}$ & 6 \\
\hline 1103 & 134 & $<1\}$ & 1 & & & 06 & $\{3111$ & $3\}$ & h \\
\hline 1.103 & $\{0111$ & $2\}$ & & & & 0.8180 & 330 & 13 & 4 \\
\hline 1.082 & 0210 & $\ll 1$ & $<1$ & & & 0.8114 & 4010 & $<1$ & $\ll 1$ \\
\hline 1.059 & $\left\{\begin{array}{l}40 \overline{1} \\
\text {. }\end{array}\right.$ & $\ll 1\}$ & $<1$ & & & & & & \\
\hline & $\{226$ & $<1\}$ & & & & & & & \\
\hline
\end{tabular}


Table 3

SIMULATED POWDER DIFFRACTION DATA FOR $\beta$-RHOMBOHEDRAL BORON

\begin{tabular}{|c|c|c|c|c|c|c|c|c|c|c|c|}
\hline \multirow[b]{2}{*}{$\begin{array}{c}\text { d-Spacings } \\
\text { (A) }\end{array}$} & \multirow[b]{2}{*}{ HKL } & \multicolumn{2}{|c|}{$\begin{array}{c}\text { CuKa } 1-2 \\
\text { Relative Intensities } \\
\text { Fiat Absorption }\end{array}$} & \multicolumn{2}{|c|}{$\begin{array}{c}\text { CrKa1-2 } \\
\text { Relative Intensities } \\
\text { Flat Absorption } \\
\end{array}$} & \multirow{2}{*}{$\begin{array}{c}\text { d-Spacings } \\
\text { (A) }\end{array}$} & \multirow[b]{2}{*}{ HKL } & \multicolumn{2}{|c|}{$\begin{array}{c}\text { CuKa1-2 } \\
\text { Relative Intensities } \\
\text { Flat Absorption } \\
\end{array}$} & \multicolumn{2}{|c|}{$\begin{array}{c}\text { CrKa } a_{1-2} \\
\text { Relative Intensities } \\
\text { Flat Absorption }\end{array}$} \\
\hline & & Integrated & $\begin{array}{c}\text { Peak } \\
\text { Height }\end{array}$ & Integrated & $\begin{array}{c}\text { Peak } \\
\text { Height }\end{array}$ & & & Integrated & $\begin{array}{c}\text { Peak } \\
\text { Height }\end{array}$ & Integrated & $\begin{array}{c}\text { Peak } \\
\text { Height }\end{array}$ \\
\hline 8.806 & 101 & 10 & 10 & 10 & . 11 & 1.777 & 333 & $<1$ & $<1$ & $<1$ & $<1$ \\
\hline 7.937 & 003 & 48 & 49 & 49 & 54 & 1.771 & 422 & $<1$ & $<1$ & $<1$ & $<1$ \\
\hline 7.415 & 012 & 5 & 6 & 5 & 6 & & $\{1310$ & 1) & & 1 & 1 \\
\hline 5.472 & 110 & 16 & 16 & 16 & 16 & 1.763 & $\{5 \overline{05}$ & $7\}$ & 6 & 6 & 5 \\
\hline 5.041 & 104 & 100 & 100 & 100 & 100 & 1.756 & 238 & 8 & 6 & 8 & 5 \\
\hline 4.648 & 021 & 37 & 38 & 37 & 38 & 1.708 & 0213 & 4 & 4 & 4 & 3 \\
\hline 4.505 & 113 & 20 & 21 & 20 & 21 & 1.607 & $6 \overrightarrow{11}$ & $\because 1$ & $<1$ & 1 & $<1$ \\
\hline 4.403 & 202 & 14 & 14 & 14 & 14 & & $\{3012$ & 2) & & 2) & \\
\hline $4.2 b b$ & U1b & 15 & 15 & 15 & 15 & 1.680 & 40010 & $2\}$ & 3 & $2\}$ & 2 \\
\hline 3.968 & 006 & 11 & 11 & 11 & 10 & 1.674 & $01 \underline{14}$ & 1 & 1 & 1 & 1 \\
\hline 3.708 & 024 & 13 & 14 & 13 & 13 & 1.671 & $31 \underline{11}$ & 1 & 1 & 1 & $<1$ \\
\hline 3.542 & 211 & 20 & 20 & 20 & 19 & & $\{3 \overline{36}$ & $1)$ & & $1\}$ & \\
\hline 0.430 & 172 & 10 & 10 & 10 & 10 & 1.ศิร & $\{$ us $\}$ & $2\}$ & ? & $2\}$ & 2 \\
\hline 3.359 & 205 & 3 & 3 & 3 & 3 & 1.637 & 514 & $<1$ & $\ll 1$ & $<1$ & $\ll 1$. \\
\hline 3.213 & 116 & $<1$ & $<1$ & $<1$ & $<1$ & & 419 & $<1$ & & $1)$ & \\
\hline 3.202 & 107 & $\ll 1$ & $<1$ & $\ll 1$ & $\ll 1$ & 1.629 & $\{149$ & $<1\}$ & 1 & $<1\}$ & 1 \\
\hline 3.159 & 300 & $\ll 1$ & $\ll 1$ & $\ll 1$ & $\ll 1$ & 1.606 & 2212 & $<1$ & $<1$ & $<1$ & $<1$ \\
\hline 3.069 & 214 & 4 & 4 & 4 & 4 & 1.601 & $20 \overline{14}$ & $<1$ & $<1$ & $<1$ & $\ll 1$ \\
\hline & $\{303$ & 6) & & 6) & & 1.649 & $0 \overrightarrow{63}$ & 12 & 9 & 14 & 8 \\
\hline 2.935 & $\{033$ & $<1\}$ & 7 & $<1\}$ & 6 & 1.518 & 520 & $<1$ & $<1$ & 1 & $<1$ \\
\hline 2.863 & 125 & 16 & 16 & 15 & 13 & 1.502 & 339 & $\ll 1$ & $\ll 1$ & $<1$ & $\ll 1$ \\
\hline 2.840 & 018 & 3 & 4 & 3 & 3 & 1.477 & 158 & $<1$ & $<1$ & 1 & $<1$ \\
\hline 2.763 & 027 & 4 & 4 & 4 & 3 & 1.470 & 10.16 & $\ll 1$ & $\ll 1$ & $<1$ & $<1$ \\
\hline 2.736 & 220 & 8 & 7 & 8 & 7 & & $\{6 \overline{06}$ & $4\}$ & 4 & $6\}$ & \\
\hline 2.646 & 009 & 8 & 8 & 8 & 7 & 1.468 & ใ กตร & $<1\}$ & 4 & $<1\}$ & 4 \\
\hline 2.613 & 131 & 12 & 11 & 11 & 9 & 1.449 & 4013 & 9 & 7 & 14 & 7 \\
\hline 2.587 & 223 & 7 & 7 & 7 & 6 & & & & & & \\
\hline 2.567 & 312 & 5 & 5 & 5 & 4 & 1.44 .3 & 1 fิ1 & 2 & 2 & 3 & 2 \\
\hline 2.620 & 208 & 6 & 6 & $\epsilon$ & E & & & & & & \\
\hline & 306 & $6)$ & & 5 & & 1.435 & 612 & 1 & 1 & 2 & 1 \\
\hline 2.470 & 036 & $7\}$ & 13 & $6\}$ & 11 & & $41 \underline{12}$ & 2) & & 4) & \\
\hline & 217 & $10)$ & & 10 . & & 1.432 & $\{14 \overline{12}$ & $1\}$ & 12 & $2\}$ & 13 \\
\hline 2.405 & 134 & 10 & 10 & 10 & 8 & & $24 \underline{10}$ & 13 & & 20 & \\
\hline 2.382 & 119 & 5 & 4 & 4 & 4 & & $\{31 \overline{14}$ & 3) & & 4 & 3 \\
\hline 2.350 & 401 & 12 & 10 & 11. & 9 & 1.428 & $\{5011$ & $<7\}$ & 3 & 1 & 1 \\
\hline 2.324 & 042 & 7 & 6 & 7 & 6 & 1.420 & $02 \overline{16}$ & 5. & 5 & 7 & 4 \\
\hline 2.309 & 1010 & 1 & 1 & $<1$ & $<1$ & & $30 \underline{15}$ & $<1$ & & $<1$ ) & \\
\hline 2.301 & $31 \overline{5}$ & 2 & 2 & 2 & 1 & & $03 \overline{15}$ & 4 & & 61 & \\
\hline 2.289 & 128 & 2 & $\%$ & $\overline{2}$ & 2 & 1.418 & 526 & $i$ & 5 & 2 & 5 \\
\hline 2.253 & 226 & 4 & 4 & 4 & 3 & & 256 & 1 . & & 2) & \\
\hline 2,201 & 404 & 6 & 5 & 5 & 4 & & 4.37: & 1 & & 2 & 2 \\
\hline 2.165 & 321 & 2 & 1 & 2 & 1 & 1.405 & 164 & 2 & 2 & 4 & 2 \\
\hline 2.139 & 232 & $<1$ & $\leqslant 1$ & $\leqslant 1$ & $\leqslant 1$ & 1.3866 & $01 \underline{17}$ & 2 & 2 & 3 & 2 \\
\hline 2.121 & 045 & $<1$ & $<1$ & $<1$ & $\ll 1$ & & $\{61 \underline{15}$ & $9\}$ & & 15 & 8 \\
\hline 2.110 & 0111 & $<1$ & $<1$ & $<1$ & $<1$ & 1.383 & $\{04 \underline{14}$ & $1\}$ & 7 & 2 & 4 \\
\hline 2.080 & 137 & 4 & 3 & 3 & 2 & & $\{34 \overline{8}$ & $7\}$ & & $12\}$ & \\
\hline 2.042 & 324 & 7 & 6 & 7 & 5 & 1.380 & $\{4211$ & $<1\}$ & 6 & $2\}$ & 7 \\
\hline 2.028 & 039 & 2 & 2 & 2 & 2 & & $\{21 \overline{16}$ & $<1\}$ & & 1 & 1 \\
\hline 1.834 & 146 & $<1$ & $<1$ & $<1$ & $<1$ & 1.373 & $\{2215$ & $1\}$ & 1 & 2 & 1 \\
\hline 1.824 & 330 & 2 & 2 & 2 & 1 & 1.368 & 440 & 3 & 2 & 6 & 3 \\
\hline 1.798 & $10 \underline{13}$ & 1. & 1 & 1 & $<1$ & 356 & 609 & 3) & 3 & $7\}$ & \\
\hline 1.786 & $2 \overline{41}$ & 1 & 1 & 1 & $<1$ & 1.356 & 1069 & $<1\}$ & 3 & $\left.{ }_{1}\right\}$ & 4 \\
\hline
\end{tabular}


Table 3 (Continued)

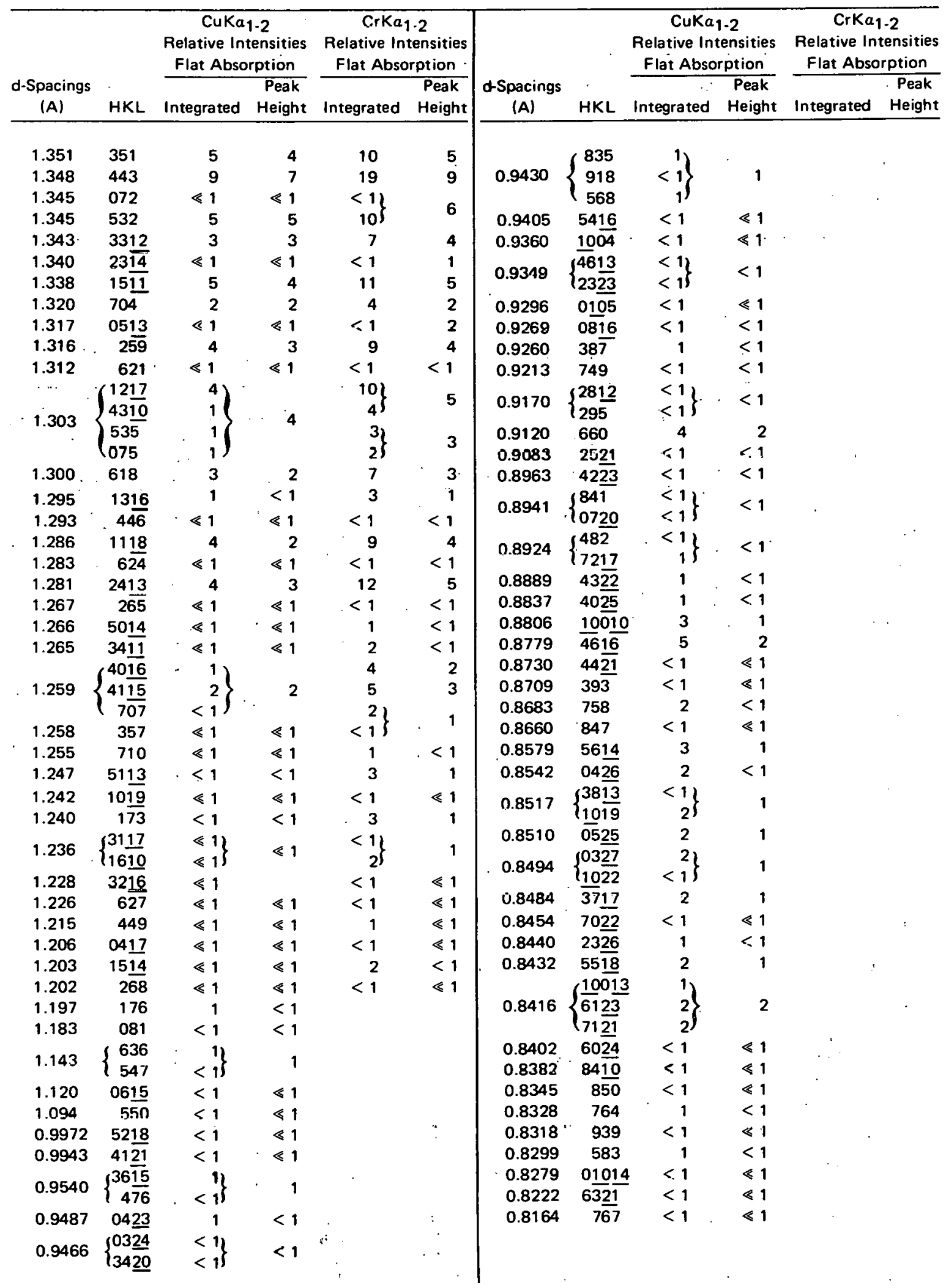


Table 3 (Continued)

\begin{tabular}{|c|c|c|c|c|c|c|c|c|c|c|c|}
\hline \multirow[b]{2}{*}{$\begin{array}{l}\text { d-Spacings } \\
\text { (A) }\end{array}$} & \multirow[b]{2}{*}{ HKL } & \multicolumn{2}{|c|}{$\begin{array}{c}\text { CuKa1-2 } \\
\text { Relative Intensities } \\
\text { Flat Absorption }\end{array}$} & \multicolumn{2}{|c|}{$\begin{array}{c}\text { CrKa1-2 } \\
\text { Relative Intensities } \\
\text { Flat Absorption }\end{array}$} & \multirow[b]{2}{*}{$\begin{array}{c}\text { d-Spacings } \\
\text { (A) }\end{array}$} & \multirow[b]{2}{*}{ HKL } & \multicolumn{2}{|c|}{$\begin{array}{l}\text { CuKa1-2 } \\
\text { Relative Intensities } \\
\text { Flat Absorption }\end{array}$} & \multicolumn{2}{|c|}{$\begin{array}{c}\text { CrKa1-2 } \\
\text { Relative Intensities } \\
\text { Flat Absorption }\end{array}$} \\
\hline & & Integrated & $\begin{array}{c}\text { Peak } \\
\text { Height }\end{array}$ & Integrated & $\begin{array}{c}\text { Peak } \\
\text { Height }\end{array}$ & & & Integrated & $\begin{array}{c}\text { Peak } \\
\text { Height }\end{array}$ & Integrated & $\begin{array}{c}\text { Peak } \\
\text { Height }\end{array}$ \\
\hline 0.8154 & $42 \underline{26}$ & $<1$ & $\ll 1$ & & & & & & & & \\
\hline 0.8133 & $\left\{\begin{array}{l}57 \overline{13} \\
26 \overline{23}\end{array}\right.$ & $\left.\begin{array}{l}<1 \\
<1\end{array}\right\}$ & $\ll 1$ & & & & & & & & \\
\hline 0.8099 & $1 \underline{115}$ & 7 & 2 & & & & & & & & \\
\hline
\end{tabular}

Table 4

SIMULATED POWDER DIFFRACTION DATA FOR A-TETRAGONAL BORON

\begin{tabular}{|c|c|c|c|c|c|c|c|c|c|c|c|}
\hline \multirow[b]{2}{*}{$\begin{array}{l}\text { d-Spacings } \\
\text { (A) }\end{array}$} & \multirow[b]{2}{*}{ HKL } & \multicolumn{2}{|c|}{$\begin{array}{l}\text { CuKa } 1-2 \\
\text { Helative Intoncitioc } \\
\text { Flat Absorption } \\
\end{array}$} & \multicolumn{2}{|c|}{$\begin{array}{c}\text { CrKa1-2 } \\
\text { Rolativa Intensiliess } \\
\text { Flat Absorption } \\
\end{array}$} & \multirow[b]{2}{*}{$\begin{array}{c}\text { d-Spacings } \\
\text { (A) }\end{array}$} & \multirow[b]{2}{*}{ HKL } & \multicolumn{2}{|c|}{$\begin{array}{l}\text { CuK } a_{1} \cdot 2 \\
\text { Kelàtive Intensities } \\
\text { Flat Absorption } \\
\end{array}$} & \multicolumn{2}{|c|}{$\begin{array}{c}\text { CrKä1-') } \\
\text { Relative Intensities } \\
\text { Flat Absorption } \\
\end{array}$} \\
\hline & & Integrated & $\begin{array}{c}\text { Peak } \\
\text { Height }\end{array}$ & Integrated & $\begin{array}{c}\text { Peak } \\
\text { Height }\end{array}$ & & & Integrated & $\begin{array}{l}\text { Peak } \\
\text { Height }\end{array}$ & Integrated & $\begin{array}{l}\text { Peak } \\
\text { Height }\end{array}$ \\
\hline 6.180 & 110 & 12 & 11 & 12 & 13 & 1.306 & 333 & 8 & 5 & 18 & 9 \\
\hline 4.370 & $\left\{\begin{array}{l}101 \\
0\end{array}\right.$ & 9 & 100 & 9) & 100 & 1.267 & 004 & 4 & 2 & 12 & 5 \\
\hline 3.919 & $\begin{array}{r}200 \\
111\end{array}$ & $\begin{array}{c}100 ! \\
72\end{array}$ & 65 & $\begin{array}{c}1005 \\
72\end{array}$ & 69 & 1.263 & 002 & 1 & 1 & 3 & 2 \\
\hline 3.095 & $\{211$ & 9 & 9 & 9) & 9 & 1.250 & 612 & 5 & 3 & 17 & 7 \\
\hline 3.095 & $\{220$ & $2\}$ & $\mathbf{y}$ & $2\}$ & $\boldsymbol{y}$ & 1.236 & 550 & $<1$ & $\ll 1$ & 3 & 1 \\
\hline 2.764 & 310 & 3 & 2 & 2 & 2 & 1.217 & 204 & $<1$ & $<1$ & 4 & 2 \\
\hline & & & & & & 1.215 & 503 & 2 & 2 & 7 & 3 \\
\hline 2.534 & 002 & 26 & 23 & 24 & 20 & 1.213 & 622 & 3 & 3 & 14 & 6 \\
\hline 2.526 & 301 & 9 & 10 & 9 & 9 & 1.212 & $\left\{\begin{array}{l}701 \\
640\end{array}\right.$ & $\left.\begin{array}{l}1 \\
2\end{array}\right\}$ & 2 & $\left.\begin{array}{l}5 \\
7\end{array}\right\}$ & 4 \\
\hline 2.426 & 311 & 83 & 70 & 78 & 66 & 1.205 & 214 & $<1$ & $<1$ & 3 & 1 \\
\hline 2.192 & 202 & 3 & 3 & 3 & 3 & 1.203 & 513 & $\leqslant 1$ & $<1$ & 1 & $<1$ \\
\hline 2.187 & 321 & $2 n$ & 17 & 18 & 15 & 1.202 & $34 \%$ & $\ll 1$ & $<1$ & 2 & $<1$ \\
\hline & & & & & & 1.201 & 711 & $\ll 1$ & $\ll 1$ & $<1$ & $\ll 1$ \\
\hline 2.060 & 330 & $<1$ & $<1$ & $<1$ & $<1$ & 1.172 & 224 & $<1$ & $\ll 1$ & 4 & 1 \\
\hline 1.956 & $\{411$ & $8\}$ & 7 & 8) & 6 & 1.170 & 523 & $\ll 1$ & $\ll 1$ & 1 & $\ll 1$ \\
\hline 1.9000 & $\{420$ & $<1\}$ & $\gamma$ & $<1\}$ & $b$ & 1.168 & 721 & $\ll 1$ & $\ll 1$ & 3 & $<1$ \\
\hline 1.908 & 331 & 8 & 6 & 8 & 6 & 1.159 & 632 & 3 & 2 & 33 & 6 \\
\hline 1.868 & 312 & 1 & $<1$ & 1 & $<1$ & 1.152 & 314 & $\Leftrightarrow 1$ & $<1$ & & \\
\hline 1.752 & 322 & 1 & $<1$ & 1 & $<1$ & 1.148 & 730 & $\ll 1$ & $<1$ & & \\
\hline 1.655 & 402 & 1 & 2 & 1 & 1 & 1.096 & 404 & 1 & $<1$ & & \\
\hline 1.653 & БU1 & 5 & 4 & 6 & 4 & 1.085 & 722 & 1 & $<1$ & & \\
\hline 1.630 & 113 & 4 & 3 & 4 & 3 & 1.026 & 434 & 2 & 1 & & \\
\hline 1636 & 112 & 4 & 4 & 5 & 3 & 1.019 & 514 & $<\overline{1}$ & $<1$ & & \\
\hline & & & & & & 1.003 & 831 & $<1$ & $\ll 1$ & & \\
\hline 1.624 & 511 & $<1$ & 1 & $<1$ & $<1$ & 0.9987 & 524 & 2 & $\lessdot 1$ & & \\
\hline 1.551 & 213 & $<1$ & $<1$ & 1 & $<1$ & 0.9962 & 751 & $<1$ & $<1$ & & \\
\hline 1.646 & $\left\{\begin{array}{l}521 \\
440\end{array}\right.$ & $\left.\begin{array}{l}3 \\
4\end{array}\right\}$ & ג & $\left.\begin{array}{l}3 \\
5\end{array}\right\}$ & 4 & 0.8787 & आय & $<1$ & $<1$ & & \\
\hline 1.457 & 600 & 6 & 4 & 9 & 5 & $\begin{array}{l}0.9652 \\
0.9595\end{array}$ & $\begin{array}{l}910 \\
841\end{array}$ & $\begin{array}{l}3 \\
1\end{array}$ & $<1$ & & \\
\hline 1.441 & 313 & 25 & 18 & 38 & 21 & 0.9560 & 604 & 2 & $<1$ & . & \\
\hline 1.439 & 432 & 13 & 11 & 20 & 12 & 0.9538 & 901 & 1 & $<1$ & & \\
\hline 1.420 & 512 & $<1$ & $\ll 1$ & $<1$ & $<1$ & 0.9503 & 614 & $<1$ & $\ll 1$ & & \\
\hline 1.382 & $\left\{\begin{array}{l}611 \\
620\end{array}\right.$ & $\left.\begin{array}{r}9 \\
12\end{array}\right\}$ & 13 & $\left.\begin{array}{l}15 \\
20\end{array}\right\}$ & 16 & $0: 8181$ & $\left\{\begin{array}{l}832 \\
911\end{array}\right.$ & $\left.\begin{array}{r}1 \\
2 \\
2\end{array}\right\}$ & $<1$ & & \\
\hline 1.367 & 522 & 14 & 9 & 26 & 13 & 0.9339 & 624 & 2 & $<1$ & & \\
\hline 1.333 & 621 & $\ll 1$ & $\leqslant 1$ & $<1$ & $\ll 1$ & 0.9318 & 761 & 1 & $<1$ & & \\
\hline 1.321 & 413 & 2 & 2 & 3 & 2. & 0.9144 & 415 & 2 & $<1$ & & \\
\hline 1.319 & 442 & $\ll 1$ & $\ll 1$ & $<1$ & 3 & 0.9113 & 851 & 2 & $<1$ & r. & \\
\hline 1.318 & 541 & 14 & 9 & 33 & 15 & 0.9095 & 335 & 1 & $<1$ & & \\
\hline
\end{tabular}


Table 4 (Continued)

\begin{tabular}{|c|c|c|c|c|c|c|c|c|c|c|c|}
\hline \multirow[b]{2}{*}{$\begin{array}{c}\text { d-Spacings } \\
\text { (A) }\end{array}$} & \multirow[b]{2}{*}{ HKL } & \multicolumn{2}{|c|}{$\begin{array}{c}\text { CuKa } 1-2 \\
\text { Relative Intensities } \\
\text { Flat Absorption }\end{array}$} & \multicolumn{2}{|c|}{$\begin{array}{c}\text { CrKa } a_{1-2} \\
\text { Relative Intensities } \\
\text { Flat Absorption }\end{array}$} & \multirow[b]{2}{*}{$\begin{array}{l}\text { d-Spacings } \\
\text { (A) }\end{array}$} & \multirow[b]{2}{*}{ HKL } & \multicolumn{2}{|c|}{$\begin{array}{c}\text { CuKa } 1-2 \\
\text { Relative Intensities } \\
\text { Flat Absorption } \\
\end{array}$} & \multicolumn{2}{|c|}{$\begin{array}{c}\text { CrKa1-2 } \\
\text { Relative Intensities } \\
\text { Flat Absorption } \\
\end{array}$} \\
\hline & & Integrated & $\begin{array}{l}\text { Peak } \\
\text { Height }\end{array}$ & Integrated & $\begin{array}{c}\text { Peak } \\
\text { Height }\end{array}$ & & & Integrated & $\begin{array}{l}\text { Peak } \\
\text { Height }\end{array}$ & Integrated & $\begin{array}{l}\text { Peak } \\
\text { Height }\end{array}$ \\
\hline $\begin{array}{l}0.9064 \\
0.9020\end{array}$ & $\begin{array}{l}931 \\
912\end{array}$ & $\left\{\begin{array}{r}1 \\
<1\end{array}\right.$ & $\begin{array}{l}<1 \\
\ll 1\end{array}$ & $\because$ & . & 0.8450 & $\left\{\frac{1021}{006}\right.$ & $\left.\begin{array}{l}3 \\
2\end{array}\right\}$ & 2 & & \\
\hline 0.8978 & 823 & $<1$ & $\$ 1$ & & & 0.8396 & 535 & 1 & $<1$ & & \\
\hline 0.8879 & $\left\{\begin{array}{l}922 \\
762\end{array}\right.$ & $\left.<\begin{array}{r}1 \\
2\end{array}\right\}$ & $<1$ & & . & 0.8380 & $\begin{array}{l}913 \\
942\end{array}$ & $\left.\begin{array}{l}7 \\
3\end{array}\right\}$ & 3 & & \\
\hline $\begin{array}{l}0.8829 \\
0.8769\end{array}$ & $\begin{array}{l}770 \\
505\end{array}$ & $\begin{array}{l}1 \\
2\end{array}$ & $\begin{array}{r}<1 \\
1\end{array}$ & & & $\begin{array}{l}0.8337 \\
0.8293\end{array}$ & $\begin{array}{l}772 \\
206\end{array}$ & $\begin{array}{r}<1 \\
2\end{array}$ & $\begin{array}{l}\ll 1 \\
<1\end{array}$ & & \\
\hline 0.8758 & 644 & 1 & $<1$ & & & 0.8282 & 615 & 2 & $<1$ & & \\
\hline $\begin{array}{l}0.8750 \\
0.8715\end{array}$ & $\begin{array}{l}833 \\
724\end{array}$ & $\begin{array}{r}<1 \\
2\end{array}$ & $\begin{array}{l}\ll 1 \\
<1\end{array}$ & . &. & 0.8259 & $\left\{\frac{1031}{216}\right.$ & $\left.\begin{array}{r}1 \\
1 \\
1\end{array}\right\}$ & $<1$ & & \\
\hline 0.8707 & 753 & 2 & 1 & & & 0.8237 & 814 & $<1$ & $\ll 1$ & & \\
\hline 0.8701 & 852 & 4 & 2 & & & 0.8173 & 625 & $<1$ & $\ll 1$ & & \\
\hline 0.8597 & 525 & 2 & $<\overrightarrow{1}$. & & & 0.8138 & 545 & 6 & 2 & & \\
\hline 0.8506 & 734 & $<1$ & $\ll 1$ & & & 0.8116 & 871 & $<1$ & $\ll 1$ & & \\
\hline 0.8489 & 050 & 3 & 1 & & & 0.8088 & 933 & 6 & 2 & . & \\
\hline 0.8459 & 843 & 3 & 1 & & & & & & & & \\
\hline
\end{tabular}

The reflections listed in the tables are those with a relative integrated intensity greater than or equal to 0.5 , with the exception of smaller reflections that had been previously reported in the literature. The lines listed as having an intensity value $\ll 1$ have a calculated relative value between 0 and. 0.4 ; those with a value of $<1$ have a relative value between 0.5 and 0.9. All other relative intensity values are rounded to the nearest whole value.

Agreement between the calculated peak height intensities of $a$-rhombohedral boron (Table 2) and those of a previously reported powder pattern is excellent. Minor discrepancies occurred among the observed pattern, $(19)$ the previously reported calculated pattern, $(6)$ and the results obtained in the present calculation. The differences are believed to be due to impurities, or preferred orientation in the sample in the case of the observed pattern and positional parameter discrepancies in the case of the previously calculated pattern. The calculated pattern of a-tetragonal boron is in good agreement with a previously published calculated pattern.(6) The pattern generated in the present study, however, is calculated through a d-spacing value of 0.8088 , thereby adding a significant number of proposed reflections to the previously generated pattern.

Calculated patterns reveal the major contributing reflections in overlapping peaks and identify high angle lines for lattice parameter refinements. Theoretical patterns are needed for detecting the intensity variation caused by solid solution formation. They also verify that the program can produce results based on good input: structure data that are comparable to experimentally observed patterns, as shown in the case of $a$-rhombohedral boron. 


\section{REFERENCES}

(1) Decker, B. F. and Kasper, J. S.; Acta Cryst, 12, p 503 (1959).

(2) Hoard, J. L., Sullenger, D. B., Kennard, C.H. L., and Hughes, R..E.; J Solid State Chem, 1, p 268 (1970).

(3) Hoard, J. L., Hughes, R. E., and Sands, D. E.; J Amer Chem Soc, 80, p 4507 (1958).

(4) Talley, C. P., LaPlaca, S.; and Post, B.; Acta Cryst, 13, p 271 (1960).

(5) Runow, P.; J Mat/s Sci, 7, p 499 (1972).

(6) Hoard, J. L. and Newkirk, A. E.; J Amer Chem Soc, 82, p 70 (1960).

(7) Sullenger, D. B.; AEC Research and Development Report MLM-1633; Monsanto Research Corporation, Mound Laboratory, Miarnlsburg, Ohio (1969). Available from the National Technical Information Service, Springfield, Virginia 22151.

(8) Matkovich, V. I.; J Amer Chem Soc, 83, p 1804 (1961).

(9) LaPlaca, S. and Post, B.; Planseeber Pulvermet, 9, p 109 (1961).

(10) Economy, J., Matkovich, V. I., and Giese, R. F.; Z Kristallogr, 122, p 248 (1965).

(11) Magnusson, B. and Brosset, C.; Acta Chem Scand, 16, p 449 (1962).

(12) Giese, R. F., Economy, J., and Matkovich, V. I.; Z Kristallogr, 122, p 144 (1965).

(13) Carlsson, J. O. and Lundstrom, T., I Less Common Metols, 22, p 317 (1970).

(14) Anderson, S. and Lundstrom; T.; J Solid State Chem, 2, p 603 (1970).

(15) Matkovich, V. I., Giese, R. F., and Economy, J.; Z Kristallogr, 122, p 116 (1965).

(16) Holcombe, C. E.; Formation of Solid Solutions of the Metals of Group II B with Boron, and the Deformation Mechanișms. Dis/orations, and Twinning in Boron. Y-1691; Union Carbide Corporation-Nuclear Division, Oak Ridge Y-12 Plant, Oak Ridge, Tennessee; October 24, 1969. Availạhle from National Tcchnical Information Service, Springfield, Virginia 22151.

(17) Smith, D: K.; University of. California, Lawrence Radiation Laboratory Report UCRL-50264; Livermore, California (1967).

(18) Smith, D. K.; Nore/co Reporter, 15, pp 57-65, 76 (1968). Published by Phillips Electronic Instruments, Mount Vernon, New York.

(19) Amendola, A.; Powder Diffraction File Card 11-618 (1959). Joint Committee of Powder Diffraction Standards, 1601 Park Lane, Swarthmore, Pennsylvania. 


\section{DISTRIBUTION}

\section{Atomic Energy Commission - ORO}

Hickman, H. D.

Zachry, D. S., Jr

\section{Lawrence Livermore Laboratory}

Crowley, W. B.

Gamble, D. P.

Hodges, A. J./Hanson, E. R:

Hoenig, C.

Kane, J. S.

Krikorian, O. H.

Kury, J. W.

Maimoni, A.

Perlman, $T$.

Stout, N. D.

Tech Inf Div

\section{Los Alamos Scientific Laboratory}

Baker, R. D.

Byers, D.

Dunn, F.

Horpedahl, L. C.

Nutt, A. W.

Olwin, R.

Schreffler, R. G.

Taub, J. M.

Taylor, J.

Wechsler, J. J.

Oak Ridge Gaseous Diffusion Plant

Wilcox, W. J., Jr

Winkel, R. A.

Oak Ridge Y-12 Plant

Bernander, N. K.

Briscoe, O. W.

Burditt, R. B.

Burkhart, L. E.

Denny, A. (2)
Dodson, W. H.

Duerkson, W. K.

Hemphill, L. F.

Holcombe, C. E., Jr (10)

Johnson, D. H.

Jones, F. W.

Keith, Alvin

Kite, H. T.

Marrow, G. B.

McLendon, J. D.

Mitchel, G. W.

Powell, G. L.

Rowan, J. H.

Srhreyer, J. M.

Smith, R. D.

Tench, F. M.

Tewes, W. E.

Trotter, T. C.

Weathersby, W. E.

Williams, R. D.

Yaggi, W. J.

$Y$-12 Central Files (5)

$Y-12$ Central Files (master copy)

$Y-12$ Central Files (route)

$\mathrm{Y}$-12 Central Files (Y-12RC)

Paducah Gaseous Diffusion Plant

Levin, R. W.

\section{Sandia-Albuquerque}

Berry, L. M.

Bild, C. F.

Braasch, R. H.

Chabai, A. J.

Gardner, W. H.

Lang, J. J.

Mail Serv Sec

McDonald, J. E.

Peurifog, R. L., Jr

Schuster, D. M.

Shuster, D. B.

Stoller, H. M. 


\section{Sandia-Livermore}

Adolphson, D. R.

Barham, J. F.

Baroody, R. A.

Brandvold, G. E.

Brierly, J. M.

Gilson, J.

Gregson, D. E.

King, J. C.

Mar, R. W.

Meinken, R. H.

Kychnovsky, R. E.

Sitiuler, G. L.

The Joint Committee on Powder Diffraction Standards (Swarthmore, Pa)

The Pennsylvania State University-Materials Research Laboratory

Spear, K. E.

In addition, this report is distributed in accordance with the category UC-4, Chemistry, as given in the USAEC Standard Distribution Lists for Unclassified Scientific and Technical Reports, TID-4500. 